\title{
ALMACENAMIENTO DE CARBONO EN INDIVIDUOS DE CAMU CAMU ARBUSTIVO [Myrciaria dubia (H.B.K) Mc Vaugh] PLANTADOS EN EL CENTRO EXPERIMENTAL SAN MIGUEL, IQUITOS, PERÚ
}

\author{
Cynthia DIAZ-CORDOVA', Enrique LOPEZ-LAVAJOS ${ }^{1}$, Jhon DEL AGUILA-PASQUEL ${ }^{2}$, Elvis \\ PAREDES-DAVILA ${ }^{2}$, Mario PINEDO-PANDURO ${ }^{2}$, Carlos ABANTO-RODRIGUEZ ${ }^{2}$ \\ 1 Universidad Nacional de la Amazonia Peruana, Facultad de Ingeniería Forestal, Escuela de Ingeniería de Bosques \\ Tropicales, Iquitos, Perú. E-mail cynelidc@hotmail.com \\ 2 Instituto de Investigaciones de la Amazonia Peruana, Av. A. Quiñones km 2,5 Iquitos, Perú. E-mail: \\ jdelaguilap@iiap.org.pe
}

\section{RESUMEN}

Este estudio tiene como objetivo estimar el stock de carbono almacenado en individuos de camu camu arbustivo Myrciaria dubia, plantados en el Centro Experimental San Miguel (CESM), Loreto, Perú. Se seleccionaron aleatoriamente 10 individuos de una plantación de 13 años de edad, perteneciente a un banco de germoplasma que representa cinco cuencas de la Amazonía peruana. Estos individuos fueron sometidos a muestreo destructivo, es decir, se taló el arbusto, se obtuvo el peso fresco de cada estructura vegetal (hojas, tallo y raíces) y se llevaron al laboratorio muestras representativas para obtener el contenido de humedad y la densidad específica de la madera. El stock de carbono promedio en las hojas, tallo y raíces fue $0.84,18.67$ y $3.05 \mathrm{~kg}$ respectivamente. Mientras que el contenido de humedad fue 39.32, 34.60 y $31.64 \%$ para hojas, raíces y tallo respectivamente. La densidad específica de la madera varió de 0.75 a $0.80 \mathrm{~g} \mathrm{~cm}^{-3}$. La densidad de carbono estimada para la plantación es mayor $\left(102.02 \mathrm{t} \mathrm{C} \mathrm{ha}^{-1}\right)$ a lo reportado para otras plantaciones de arbustos amazónicos como el "cacao" y el "copoazú". Estos valores ayudarán a generar un modelo alométrico que ayudará a una mejor estimación del stock de carbono de plantaciones de camu camu en la Amazonía.

PALABRAS CLAVE: Myrciaria dubia, stock de carbono, humedad, densidad específica de la madera.

\section{CARBON STORAGE OF PLANTED INDIVIDUALS OF THE SHRUBBY "CAMU CAMU" [Myrciaria dubia (H.B.K) Mc Vaugh] IN THE SAN MIGUEL EXPERIMENTAL CENTER, IQUITOS, PERU}

\begin{abstract}
This study aims to estimate the carbon stock of shrubby "camu camu" individuals planted in the San Miguel Experimental Centre, Loreto, Peru. We randomly selected 10 individuals of a 13-year-old plantation belonging to a gene bank that represent five Peruvian Amazon basins. These individuals were destructively sampled, i.e. the shrub was cut, , fresh weight was recorded for each plant structure (leaves, stem and roots), and representative samples were taken to the lab to get the moisture content and the specific wood density. The average carbon stock in leaves, stem and roots was $0.84,18.67$ and $3.05 \mathrm{~kg}$, respectively. While the moisture content was $39.32,34.60$ and $31.64 \%$ for leaves, roots and stem respectively. The specific wood density varied from 0.75 to $0.80 \mathrm{~g} \mathrm{~cm}^{-3}$. The estimated carbon stock for the whole plantation is higher $(102.02 \mathrm{t}$ $\left.\mathrm{C} \mathrm{ha-}^{1}\right)$ than those values reported for other Amazonian shrub plantations like "cacao" and "copazú". These values will help to generate an allometric model that better estimate the carbon stock of camu camu plantations in the Amazon.
\end{abstract}

KEYWORDS: Myrciaria dubia, carbon stock, moisture content, specific wood density. 


\section{INTRODUCCIÓN}

A nivel mundial se hacen cada vez más evidentes los efectos del cambio climático, observándose oleadas de frío y de calor cada vez más severas y más frecuentes a lo acostumbrado. Estos cambios se atribuyen principalmente al aumento de las concentraciones de gases de efecto invernadero (GEI) en la atmósfera, tales como el dióxido de carbono $\left(\mathrm{CO}_{2}\right)$ y metano $\left(\mathrm{CH}_{4}\right.$, IPCC, 2007). Ambas moléculas forman parte del ciclo biogeoquímico de carbono por lo que su mejor entendimiento se ha convertido en un tema importante en la agenda ambiental global (Gay \& Martínez, 1995).

En los bosques tropicales existen varios métodos desarrollados para la cuantificación de los stocks y flujos de carbono del bosque (Marthews et al., 2014; Honorio \& Baker, 2010; Kauffman \& Donato, 2012). En el Perú, específicamente en la Amazonia baja, la mayoría de estudios desarrollados por entidades peruanas se basan en stocks de carbono de bosques primarios (Freitas et al., 2006; García et al., 2012) y plantaciones forestales (Martínez, 2002; Rojas, 2004). Sin embargo, los estudios orientados hacia el stock de carbono en arbustos (rodales o plantaciones) son escasos y solo se centran en algunas especies como el cacao Theobroma cacao y copoazú Theobroma grandiflorum (Martínez et al., 2013; Concha et al., 2007). Hasta el momento no existe información previa sobre el stock de carbono de plantaciones de camu camu arbustivo Myrciaria dubia, que es un arbusto amazónico conocido por poseer frutos con los más altos contenidos de vitamina $\mathrm{C}$ y por ende ser ampliamente comercializado (Imán et al., 2011).

La forma primaria de cuantificar el stock de carbono de una planta (arbusto o árbol) se basa en obtener su peso seco o biomasa (Martinez et al., 2013). Asimismo a partir del peso seco y registrando las variables dasométricas básicas (diámetro, altura, entre otros) de un número mínimo de individuos es posible generar una ecuación alométrica que permita estimar el carbono almacenado en la planta (Picard et al., 2012).

En ese sentido, este trabajo de investigación se planteó cuantificar los stocks de carbono de las estructuras vegetales (hojas, tallo y raíces) del camu camu arbustivo Myrciaria dubia. Además de conocer algunas de sus características básicas, como el contenido de humedad y la densidad específica de madera del tallo. La información generada podrá ser utilizada como insumo básico para el diseño de ecuaciones alométricas que permitan estimar de manera precisa y eficiente el stock de carbono aéreo almacenado en la biomasa del camu camu arbustivo.

\section{MATERIAL Y MÉTODOS}

\section{Área de estudio}

El estudio fue realizado en el Centro Experimental San Miguel (CESM) del Instituto de Investigaciones de la Amazonía peruana (IIAP), ubicado en el departamento de Loreto (distrito de Belén al margen izquierdo del rio Amazonas), aguas arriba de la desembocadura del rio Itaya (coordenadas: $3^{\circ} 40^{\prime} \mathrm{S}, \quad 73^{\circ} 10^{\prime} \mathrm{O}$ ). El área está compuesta por un complejo de orillares del río Amazonas, el cual presenta diques y bacines, denominados vernacularmente como restinga $y$ bajiales. Esta zona se encuentra constituida por depósitos fluviales, pertenecientes al cuaternario (Qfr) afectado por inundaciones periódicas ocasionadas por la creciente del rio Amazonas. Litológicamente está compuesta por material abundante de arcilla, y pequeñas proporciones de limo y arena (Inga \& Pinedo, 2000). El clima es cálido húmedo, la temperatura promedio es de $26^{\circ} \mathrm{C}$ y la precipitación pluvial anual es de $2911.7 \mathrm{~mm}$ año ${ }^{1}$ (Pinedo, 2002).

\section{Zona de muestreo y análisis}

El estudio se desarrolló en una plantación sobre restinga alta de 13 años de edad, con una extensión de $90 \mathrm{~m}$ x $50 \mathrm{~m}$ y con una densidad de siembra de 1.5 m x $1 \mathrm{~m}$, la cual está conformada por 2035 arbustos procedentes de cinco cuencas amazónicas (Itaya, Tigre, Napo, Curaray y Putumayo). Forma parte del banco de Germoplasma de ecotipos de camu camu arbustivo del proyecto "Mejoramiento genético de camu camu arbustivo Myrciaria dubia manejado por el IIAP y el Instituto Nacional de Investigación Agraria y Agroindustrial(INIA).

El laboratorio donde se realizó los análisis de las muestras está ubicado en la Estación Experimental Agraria San Roque del INIA, a una distancia de 7 $\mathrm{km}$ de la ciudad de Iquitos y tiene como coordenadas $03^{\circ} 45^{\prime} 05^{\prime \prime} \mathrm{S}, 73^{\circ} 14$ ' $40^{\prime \prime} \mathrm{O}$.

\section{Determinación del stock de carbono y contenido de humedad}

Fueron seleccionados de manera aleatoria un total de 10 individuos de la plantación. De cada individuo fueron colectados el total de hojas y de raíces, estas últimas fueron colectadas talando el individuo al ras del suelo y finalmente cavando el suelo para extraer las raíces gruesas y finas del arbusto. Las tres estructuras vegetales: hojas, tallo (y ramas) y raíces fueron separadas y colocadas en costales para ser pesadas. Se registró el peso fresco 
en campo y luego se tomó una muestra de $1 \mathrm{~kg}$ de las estructuras, la cual fue empaquetada, debidamente rotulada y llevada al laboratorio.

En el laboratorio las muestras fueron colocadas en sobres manila y puestas a secar en una estufa eléctrica a $95^{\circ} \mathrm{C}$ por un período aproximado de una semana hasta obtener un peso constante. Finalmente se registró el peso seco en laboratorio.

Para calcular el stock de carbono se obtuvo el contenido de humedad de las muestras de cada estructura llevadas al laboratorio.

$$
C H=\frac{\left(P_{f}-P_{s}\right)}{P_{f}} \times 100
$$

Dónde:

CH es contenido de humedad (\%)

$\mathbf{P}_{\mathrm{f}}$ es peso fresco $(\mathrm{g})$

$\mathbf{P}_{\mathrm{s}}$ es peso seco $(\mathrm{g})$

Después se aplicó la siguiente fórmula para calcular el stock de carbono en la estructura (hojas, tallo y raíces) que corresponda.

$$
C=\frac{P_{f c}-\left(\frac{C H_{m}}{100} \times P_{f c}\right)}{2}
$$

Dónde:

C es stock de carbono de estructura $(\mathrm{kg})$

$\mathbf{P}_{\mathrm{fc}}$ es peso fresco total en campo $(\mathrm{kg})$

$\mathbf{C h}_{\mathrm{m}}$ es contenido de humedad por muestra (\%)

Finalmente, para conocer el stock de carbono total de cada individuo se sumaron los valores calculados de cada una de sus estructuras.

\section{Densidad específica de madera}

Por cada tallo de los individuos seleccionados se extrajo una muestra pequeña de madera, la cual fue rotulada y llevada al laboratorio. Se calculó el volumen exacto de la muestra usando el método de desplazamiento de agua (Chave et al., 2006). Luego se secó la muestra a $95{ }^{\circ} \mathrm{C}$ hasta obtener un peso constante, el cual fue registrado para la siguiente fórmula:

$$
C M=\frac{P_{s}}{V}
$$

Dónde:

DM es la densidad específica de madera $\left(\mathrm{g} \mathrm{cm}^{-3}\right)$

$\mathbf{P}_{\mathrm{s}}$ es el peso seco de la muestra $(\mathrm{g})$

V es el volumen de la muestra $\left(\mathrm{cm}^{3}\right)$

\section{RESULTADOS Y DISCUSIÓN}

\section{Stock de carbono}

El stock de carbono total de Myrciaria dubia fue $22.56 \mathrm{~kg} \mathrm{C}$ en promedio. Mientras que el valor promedio de las hojas, tallos y raíces fue de 0.84 ; 18.67 y $3.05 \mathrm{~kg} \mathrm{C}$ respectivamente (Tabla 1 y Figura 1).

El valor total de carbono por individuo obtenido en este estudio $(6.22$ a $42.88 \mathrm{~kg} \mathrm{C})$ fue mayor a lo reportado por Martínez et al. (2013) quién determinó que el stock de carbono en individuos de cacao Theobroma cacao L varió de 0.235 a $1.12 \mathrm{~kg}$ de C, y en individuos de copoazú (Theobroma grandiflorum Willd. ex Spreng., K. Schum.) estuvo entre 0.235 y $2.385 \mathrm{~kg}$ de C.

Tabla 1. Stock de carbono por estructura vegetal del camu camu arbustivo Myrciaria dubia.

\begin{tabular}{ccccc}
\hline \multirow{2}{*}{$\mathbf{N}^{\mathbf{0}}$} & \multicolumn{4}{c}{ Carbono $(\mathbf{k g})$} \\
\cline { 2 - 5 } & Hoja & Tallo & Raíz & TOTAL \\
\hline 1 & 1.63 & 33.79 & 5.36 & 40.78 \\
2 & 1.09 & 36.15 & 5.64 & 42.88 \\
3 & 1.64 & 27.24 & 3.26 & 32.14 \\
4 & 0.33 & 7.55 & 1.23 & 9.11 \\
5 & 0.96 & 21.66 & 3.36 & 25.98 \\
6 & 0.66 & 16 & 2.79 & 19.45 \\
7 & 0.73 & 22,24 & 3.21 & 26.18 \\
8 & 0.44 & 5.7 & 1.67 & 7.81 \\
9 & 0.44 & 4.38 & 1.4 & 6.22 \\
10 & 0.48 & 11.95 & 2.6 & 15.03 \\
\hline Promedio & $\mathbf{0 . 8 4}$ & $\mathbf{1 8 . 6 7}$ & $\mathbf{3 . 0 5}$ & $\mathbf{2 2 . 5 6}$ \\
\hline
\end{tabular}

Tabla 2. Contenido de humedad por estructura vegetal del camu camu arbustivo Myrciaria dubia.

\begin{tabular}{cccc}
\hline \multirow{2}{*}{$\mathbf{N}^{\mathbf{0}}$} & \multicolumn{3}{c}{ Carbono $(\mathbf{k g})$} \\
\cline { 2 - 4 } & Hoja & Tallo & Raíz \\
\hline 1 & 37.32 & 37.05 & 34.19 \\
2 & 40.83 & 34.32 & 29.87 \\
3 & 38.19 & 48.21 & 49.20 \\
4 & 39.98 & 45.93 & 49.79 \\
5 & 43.51 & 37.41 & 32.19 \\
6 & 46.90 & 39.17 & 38.76 \\
7 & 45.19 & 29.13 & 18.22 \\
8 & 34.51 & 26.45 & 22.46 \\
9 & 34.93 & 25.70 & 17.46 \\
10 & 31.81 & 22.65 & 24.23 \\
\hline Promedio & 39.32 & 34.60 & 31.64 \\
\hline
\end{tabular}




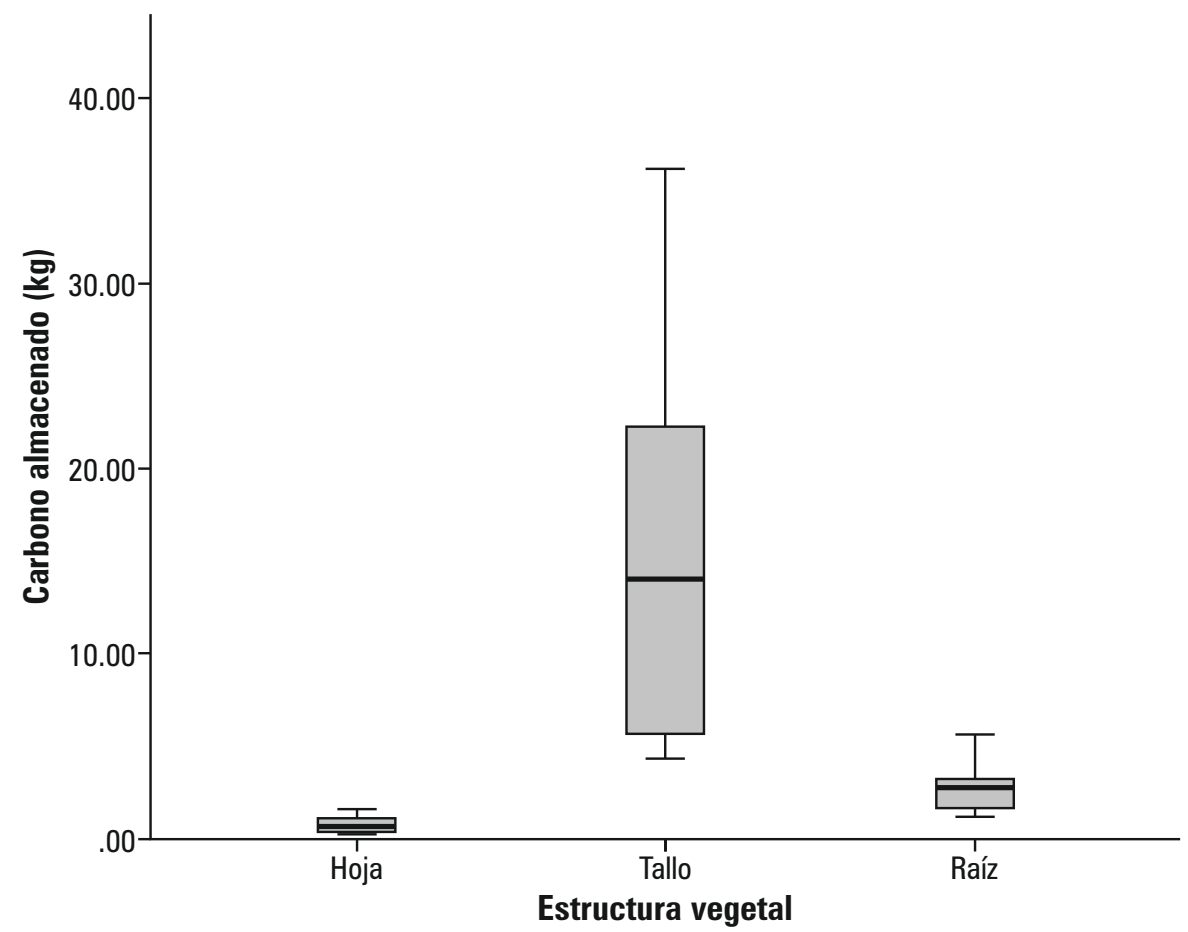

Figura 1. Valor promedio del stock de carbono en las estructuras vegetales del camu camu arbustivo Myrciaria dubia.

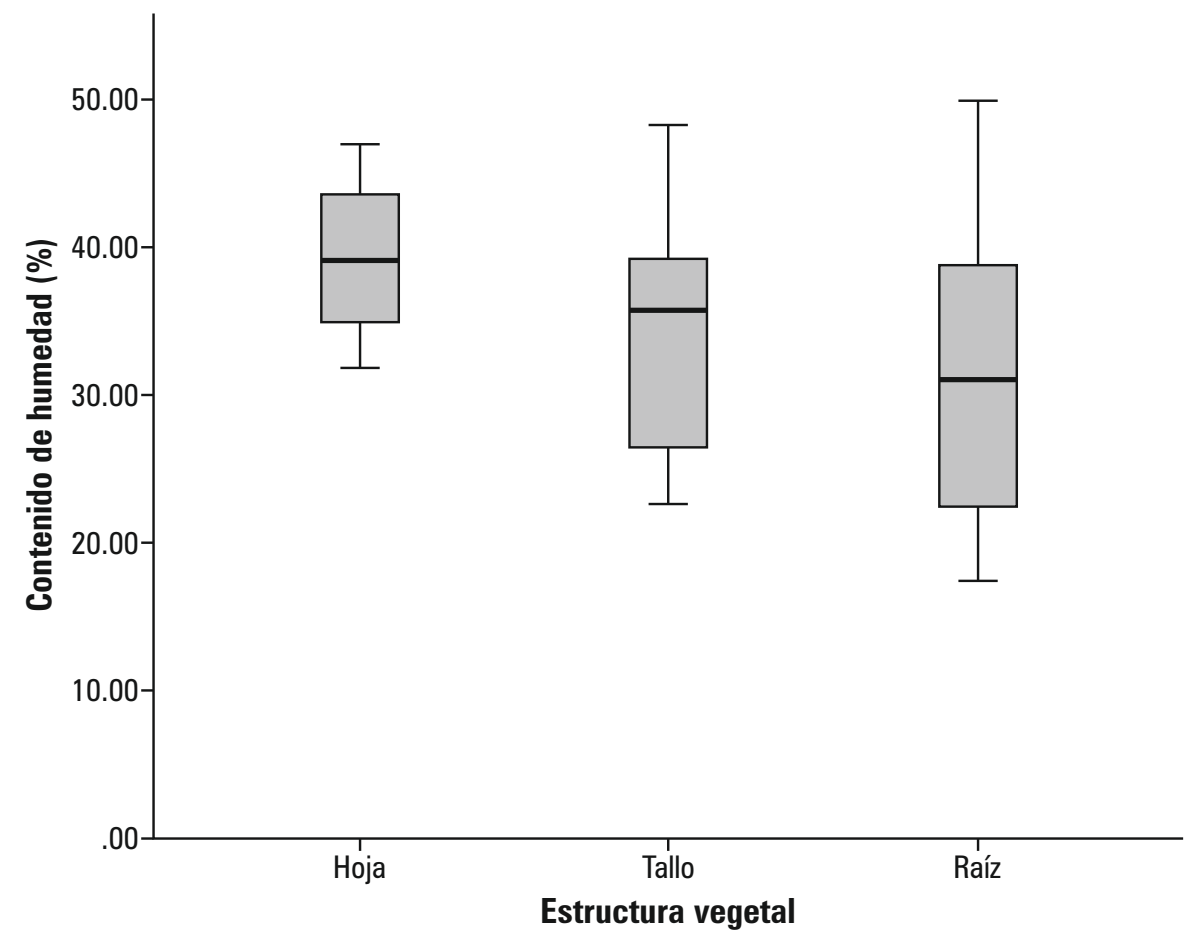

Figura 2. Porcentaje del contenido de humedad en las estructuras vegetales del camu camu arbustivo Myrciaria dubia. 


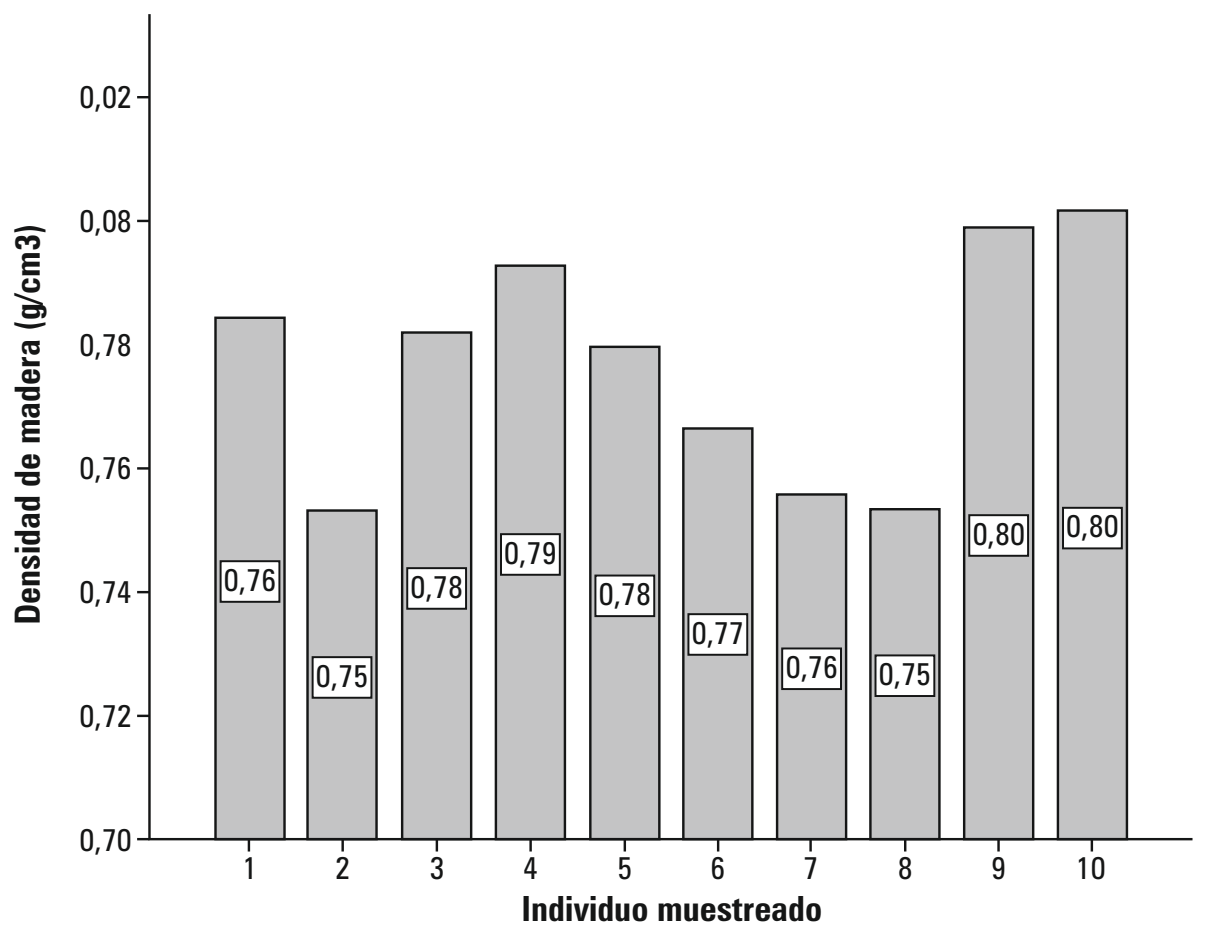

Figura 3. Densidad específica de madera por individuo del camu camu arbustivo Myrciaria dubia.

La variación encontrada en los valores de carbono total por individuo está relacionada a la heterogeneidad mostrada por las estructuras vegetales de cada individuo, es decir, algunas presentaban mayor cantidad de hojas que otras (Figura 1) lo que demuestra que el proceso de selección aleatoria cubrió las expectativas del estudio. Esta variación podría ser explicada por las transformaciones naturales o inducidas, como la erosión y el cambio de uso de la tierra, que sufren las plantaciones agroforestales (Woomer \& Palm, 1998).

Asimismo, la variación existente entre el carbono almacenado en las raíces y las hojas podría deberse a factores de entorno como la densidad de siembra (1.5 x $1 \mathrm{~m}$ para este estudio), la cual promueve la competencia por espacio y luz entre los individuos. Por otro lado, existen reportes de factores externos como las plagas que pueden afectar a los tallos y hojas de Myrciaria dubia, lo que generaría una variación en el carbono almacenado por ambas estructuras vegetales (Pinedo et al., 2010).
Al relacionar el valor promedio total de carbono por individuo con la superficie de la plantación evaluada se obtiene $102.02 \mathrm{t} \mathrm{C} \mathrm{ha}^{-1}$, estando dentro del rango reportado por Catriona (1998) para bosques secundarios (25-190 t C ha $\left.{ }^{-1}\right)$ y dentro del rango para ecosistemas de bosques tropicales que varía desde 67.5 hasta $171 \mathrm{t} \mathrm{C} \mathrm{ha}^{-1}$ (Alegre et al., 2001).

\section{Contenido de humedad}

El contenido de humedad promedio para hojas, tallos y raíces fue de 39.32, 34.60 y $31.64 \%$, respectivamente. El valor más alto en el contenido de humedad de las hojas (Figura 2) concuerda con Russo (1983), quien afirma que las hojas presentan el mayor porcentaje de humedad en comparación a las raíces y el tallo. Sin embargo, los rangos de humedad reportados por el mismo autor para hojas, tallos y raíces de árboles o arbustos (70-90; 50-80 y $60-90 \%$ respectivamente) difieren notablemente de los valores de este estudio. 


\section{Densidad específica de madera}

La densidad específica de madera promedio de Myrciaria dubia fue $0.78 \mathrm{~g} \mathrm{~cm}^{-3}$, valor que es mayor a la densidad reportada para los arbustos Quercus tinkhami C. H. Müll. $\left(0.74 \mathrm{~g} \mathrm{~cm}^{-3}\right)$ y Quercus sebifera Trel. $\left(0.68 \mathrm{~g} \mathrm{~cm}^{-3}\right)$ en Sierra de Âlvarez, México (Bárcenas-Pazos et al., 2008). Asimismo, es mayor a los valores reportados por Espina (2006) para individuos de Eucalyptus globulus Labill que variaron de $0.41 \mathrm{a} 0.65 \mathrm{~g} \mathrm{~cm}^{-3}$.

Además, los valores para Myrciaria dubia difieren de los de Zanne et al. (2009) quienes reportaron que las familias Lecythidaceae y Sapotaceae presentan las densidades específicas de madera más altas en los bosques amazónicos $(0.72$ y $0.77 \mathrm{~g} \mathrm{~cm}^{-3}$ respectivamente). Esta diferencia puede deberse al tipo de tejido especializado para la inundación que presenta Myrciaria dubia, ya que en general las especies tolerantes a la inundación parecen depositar más carbono en los tejidos aéreos que en los subterráneos (López et al., 2009).

\section{CONCLUSIONES}

Entre las estructuras vegetales de Myrciaria dubia, presenta el valor más alto de carbono con un promedio de $18.67 \mathrm{~kg}$ lo que equivale al $82.76 \%$ del total de carbono por cada individuo evaluado. Las hojas presentan el mayor contenido de humedad $(39.32 \%)$ dentro de las estructuras vegetales de Myrciaria dubia. En tanto que la densidad específica de madera promedio de Myrciaria dubia fue $0.78 \mathrm{~g}$ $\mathrm{cm}^{-3}$.

\section{AGRADECIMIENTO}

Agradecemos Raymundo Mariño y Francisco Guerrero (obreros de campo del CESM-IIAP). Al INIA, en especial al Ing. Sergio Pinedo y a la Técnica Eloísa Celis por el apoyo en la fase de laboratorio.

\section{BIBLIOGRAFIA CITADA}

Alegre, J.; Arévalo, L.; Ricse, A.; Callo-Concha, D.; Palm, C. 2001. Carbon sequestration for different land use systems in the humid tropics of Peru. (http://www.virtualcentre.org/es/ele/ conferencia2/vbconfe $7 . h t m)$

Bárcenas-Pazos, G.M.; Ríos-Villa, R.; AguirreRivera, J.R.; Juárez-Flores, B.I.; HonoratoSalazar, J.A. 2008. Composición química y densidad básica relativa de la madera de dos especies arbustivas de encino blanco de la Sierra de Álvarez, SLP, México. Madera y Bosques, 14(3):81-94.
Concha, J., Alegre, J., Pocomucha, V. 2007. Determinación de las reservas de carbono en la biomasa aérea de sistemas agroforestales de Theobroma cacao L. en el departamento de San Martín, Perú. Ecología Aplicada, 6(1-2):75-82.

Catriona, P. 1998. Actualidad Forestal Tropical. Boletín de Manejo Forestal Producido por la Organización de Maderas Tropicales para Fomentar la Conservación y el Manejo Sostenible de los Recursos Forestales tropicales en la región de América Latina y el Caribe (Japón). 31pp.

Chave, J.; Muller-Landau, H.C.; Baker, T.R.; Easidale, T.; Ter Steege, H.; Webb, C.O. 2006. Regional and phylogenetic variation of wood density across 2456 neotropical tree species. Ecological Applications, 16(6): 2356-2367.

Espina, A. 2006. Densidad básica de la madera de Eucalytus Globulus en dos sitios en Chile. Tesis para obtener el título de Ingeniero Forestal. Universidad Austral de Chile. Facultad de Ciencias Forestales, 4: 16-17.

Freitas, L.; Otarola, E.; Del Castillo, D.; Linares, C.; Martínez, P; Malca, G. 2006. Servicios ambientales de almacenamiento y secuestro de carbono del ecosistema aguajal en la Reserva Nacional Pacaya Samiria, Loreto - Perú. Documento técnico $\mathrm{N}^{\circ}$ 29. Instituto de Investigaciones de la Amazonia Peruana, Iquitos. 65pp.

García Soria, D.; Honorio Coronado, E.; Del Castillo D. 2012. Determinación del stock de carbono en aguajales de la cuenca del río Aguaytía, Ucayali - Perú. Folia Amazónica, 21 (1-2): 153-160.

Gay, C.; Martínez, M. 1995. Mitigation of emissions of greenhouse gases in Mexico. Interciencia, 20: 336-342.

Honorio Coronado, E.N.; Baker, T.R. 2010. Manual para el monitoreo del ciclo del carbono en bosques amazónicos. Instituto de Investigaciones de la Amazonia Peruana/ Universidad de Leeds. Lima. 54pp.

Imán, S.; Bravo, L.; Sotero, V.; Oliva, C. 2011. Contenido de vitamina $\mathrm{C}$ en frutos de camu camu Myrciaria dubia (H.B.K) Mc Vaugh, en cuatro estados de maduración, procedentes de la Colección de Germoplasma del INIA Loreto, Perú. Scientia agropecuaria, 2: 123-130.

Inga, H.; Pinedo, M. 2000. Macrozonificación de suelos aluviales del caserío San Miguel - Rio Amazonas. Instituto de Investigaciones de la Amazonia Peruana, Iquitos. 141pp.

IPCC, 2007: Cambio climático 2007: Informe de síntesis. Contribución de los Grupos de trabajo I, 
II y III al Cuarto Informe de evaluación del Grupo Intergubernamental de Expertos sobre el Cambio Climático [Equipo de redacción principal: Pachauri, R.K. y Reisinger, A. (directores de la publicación)]. IPCC, Ginebra, Suiza, 104pp.

Kauffman, J.B.; Donato, D.C. 2012 Protocols for the measurement, monitoring and reporting of structure, biomass and carbon stocks in mangrove forests. Working Paper 86. CIFOR, Bogor, Indonesia. 50pp.

López, O.R., 2009. Fisiología y ecología de comunidades arbóreas en hábitats inundables. Acta Biológica Panamensis, 1: 68-86.

Marthews, T.R.; Riutta, T.; Oliveras Menor, I.; Urrutia, R.; Moore, S.; Metcalfe, D.; Malhi, Y.; Phillips, O.; Huaraca Huasco, W.; Ruiz Jaén, M.; Girardin, C.; Butt, N.; Cain, R. and colleagues from the RAINFOR and GEM networks. 2014. Measuring Tropical Forest Carbon Allocation and Cycling: A RAINFOR-GEM Field Manual for Intensive Census Plots (v3.0). Manual, Global Ecosystems Monitoring network, http://gem.tropicalforests.ox.ac.uk/.

Martinez, P. 2002. Tablas de volumen y almacenamiento de carbono de Calycophyllum spruceanum (Bentham) Hooker f. ex Schumann "Capirona" en bosques aluviales. Tesis de pregrado, Universidad de la Amazonía Peruana. Facultad de Ciencias Forestales, Iquitos, Perú. 96pp.

Martínez, P.; Solignac, J.; Zarate, R.; Fachin, L.; Maco, J.; Jarama, A. 2013. Estimación de la biomasa con relaciones alométricas en dos especies de frutales: Theobroma cacao L. (cacao) y Theobroma grandiflorum (Willd Ex Spreng.) K. Schum. (copoazú). Folia Amazónica, 22: 67-75.

Picard, N., Saint-André, L., Henry, M. 2012. Manual de construcción de ecuaciones alométricas para estimar el volumen y la biomasa de los árboles: del trabajo de campo a la predicción. Las Naciones Unidas para la Alimentación y la Agricultura y el Centre de Coopération Internationale en Recherche Agronomique pour le Développement, Rome, Montpellier. 223pp.

Pinedo, M. 2002. Sistemas de plantacion y mejora genetica de camu camu arbustivo - Myrciaria dubia - Loreto, Peru. Instituto de Investigaciones de la Amazonía Peruana, Iquitos. 9pp.

Pinedo, M.; Delgado, C.; Farroñay, R.; Del Castillo, D.; Iman, S.; Villacrés, J.; Faching, L.; Oliva, C.; Abanto, C.; Bardales, R.; Vega, R. 2010. Camu camu (Myrciaria dubia, Myrtaceae) Aportes para su aprovechamiento sostenible en la Amazonía peruana. Instituto de Investigaciones de la Amazonia Peruana Iquitos. 48pp.

Rojas, T. 2004. Modelos de crecimiento y fijación de carbono en plantaciones de Cedrelinga catenaeformis D. Ducke (Tornillo) y Simarouba amara Aublet (Marupá) en plantaciones forestales de Jenaro Herrera. Tesis de pre-grado, Universidad de la Amazonía Peruana. Facultad de Ciencias Forestales, Iquitos, Perú. 124pp.

Russo, R.O. 1983. Mediciones de biomasa en sistemas agroforestales. CATIE, Turrialba, Costa Rica. 27pp.

Woomer, P.; Palm. C. 1998. An approach to estimating system carbón stocks in tropical forests and associated land uses. Commonwealth Forestry Review, 77(3): 181-190.

Zanne, A.; López, G.; Coomes, D.; Ilic, J.; Jansen, S.; Lewis, S.; Miller, R.; Swenson, N.; Wiemann, M.; Chave, J. 2009. Global wood density data b a s e. D r y a d. I d e n t i f i e r: (http://hdl.handle.net/10255/dryad.235.)

Recibido: 14 de febrero del 2015

Aceptado para publicación: 21 de abril del 2015 
